\title{
Radiative Boundary Layer Flow in Porous Medium due to Exponentially Shrinking Permeable Sheet
}

\author{
Paresh Vyas ${ }^{1}$ and Nupur Srivastava ${ }^{2}$ \\ ${ }^{1}$ Department of Mathematics, University of Rajasthan, Jaipur 302004, India \\ ${ }^{2}$ Department of Mathematics, Poornima University, Jaipur 303905, India \\ Correspondence should be addressed to Nupur Srivastava, nupur2209@gmail.com
}

Received 24 September 2012; Accepted 10 October 2012

Academic Editors: D. Bratko and Q. Zhang

Copyright (C) 2012 P. Vyas and N. Srivastava. This is an open access article distributed under the Creative Commons Attribution License, which permits unrestricted use, distribution, and reproduction in any medium, provided the original work is properly cited.

\begin{abstract}
This communication pertains to the study of radiative heat transfer in boundary layer flow over an exponentially shrinking permeable sheet placed at the bottom of fluid saturated porous medium. The porous medium has permeability of specified form. The fluid considered here is Newtonian, without phase change, optically dense, absorbing-emitting radiation but a nonscattering medium. The setup is subjected to suction to contain the vorticity in the boundary layer. The radiative heat flux in the energy equation is accounted by Rosseland approximation. The thermal conductivity is presumed to vary with temperature in a linear fashion. The governing partial differential equations are reduced to ordinary differential equations by similarity transformations. The resulting system of nonlinear ordinary differential equations is solved numerically by fourth-order Runge-Kutta scheme together with shooting method. The pertinent findings displayed through figures and tables are discussed.
\end{abstract}

\section{Introduction}

Flow and heat transfer in boundary layer flow of viscous fluid due to deforming surface is pivotal in many industrial processes cutting across different realms. Crane [1] pioneered a closed form analytical solution for an incompressible fluid flow due to a linearly stretching sheet. This novel flow configuration in fluid mechanics was well received by the investigators to extract pertinent information in real analogous systems like extrusion processes, hot rolling, wire drawing and glass wire production, and so forth, to name a few. The so-called stretching surface flow problem has been studied for variety of assumptions pertaining to surface velocity (linear/power law) and surface temperature (constant/variable)/ temperature flux for the simple reason that optimal quality of desired component squarely depends on the shear and heat transfer rate at the sheet. The problem has also been discussed for non-Newtonian fluid, Ferro-fluid and micropolar fluid and also by incorporating physical features like suction/injection, MHD, chemical reaction, and so forth [29]. Recently, Liao [10, 11] discovered multiple solutions for the flow over both impermeable and permeable stretching sheets. In order to complete the discussion, attempts were also made to explore flow and/or heat transfer due to rather unusual exponential stretching surface. Magyari and Keller [12] discussed boundary layer flow due to a sheet stretching exponentially and bearing a variable temperature of exponential fashion. Elbashbeshy [13] furnished numerical findings about flow and heat transfer over an exponentially stretching surface subjected to mass suction. Exponentially stretching surface in the presence of magnetic field was analysed by Al-Odat et al. [14]. Partha et al. [15] considered dissipative mixed convection flow past an exponentially stretching surface. The problem was also extended to viscoelastic fluids by Sanjayanand and Khan [16, 17]. Sajid and Hayat [18] examined radiative boundary layer flow due to exponentially stretching sheet and reported HAM series solution.

Looking at the literature of flow due to deforming boundary, one finds that flow due to shrinking boundary is still a relatively low explored area. Investigation of boundary layer flow and heat transfer past shrinking surfaces requires much attention as it finds important applications in many industries and manufacturing processes. A heat shrinking sheet provides a high quality field coating solution for welded pipe 
ends. A heat shrinking tape is used for wrapping junctions. Wang [19] was probably the first to report the boundary layer flow over an unsteady shrinking film. In a subsequent study, Miklavčič and Wang [20] reasoned that flow due to shrinking sheet cannot exist unless adequate suction is applied across the boundary layer to contain the vorticity. In their study they reported a pertinent finding that in shrinking flow problem one may come across multiple solutions for different suction rates. Wang [21] studied two-dimensional and axisymmetric stagnation point flow towards a shrinking sheet and concluded that solutions do not exist for larger shrinking rates. Appreciating the possibility of exploring new avenues in flow arising due to shrinking surface, some attempts have been made recently [22-29]. MHD flow and heat transfer in a channel bounded by a shrinking sheet and a plate with a porous substrate was discussed by Chauhan and Agarwal [30]. Though the work of Bhattacharyya [31] throws some light on boundary layer flow and heat transfer past an exponentially shrinking sheet, however, the study of flow dynamics past an exponentially shrinking surface in porous media is still unexplored.

All the above-said investigations regarding "shrinking surface problems" consider clear fluid region with constant thermal conductivity. The thermal conductivity is an intrinsic property of fluids which exhibits variations with temperature. Many authors have reported studies with variable thermal conductivity aspects [32-34]. Khan et al. [35] studied thin film flow over a shrinking/stretching sheet taking variable viscosity and thermal conductivity into account.

The radiative thermal regime in porous medium has drawn much attention recently due to ample applications, such as, gasification of oil shale, waste heat storage in aquifers, and so forth. To be specific, in the case of gasification, large temperature gradient exists in the vicinity of the combustion regime making radiation effect dominant. However, literature is rather scanty on the radiative flow in porous media. A few relevant texts/studies have been presented [3639]. Radiative flow of Jeffery fluid with variable thermal conductivity in a porous medium was discussed by Hayat et al. [40]. Elbashbeshy and Emam [41] discussed the effects of radiation and heat transfer over an unsteady stretching surface embedded in a porous medium.

The present study envisages the flow past an exponentially shrinking sheet placed at the bottom of fluid saturated porous medium taking variable thermal conductivity and radiation into account. It is expected that the present work would help peep into analogous real world systems.

\section{Mathematical Model}

Let us consider the steady two-dimensional boundary layer flow of optically thick viscous Newtonian fluid and associated heat transfer over a permeable sheet placed at the bottom of a fluid saturated porous medium having permeability of specific form. A Cartesian coordinate system is chosen where the $x$-axis is taken along the sheet and $y$-axis is normal to it. The flow is caused by the sheet shrinking in an exponential fashion. A suction is applied normal to sheet to contain the vorticity. The fluid considered here is without phase change, optically dense, absorbing-emitting radiation but a nonscattering medium. The thermal conductivity of the fluid is assumed to vary linearly with temperature. The radiation flux in the energy equation is presumed to follow Rosseland approximation. The boundary layer equations for the considered setup are

$$
\begin{gathered}
\frac{\partial u}{\partial x}+\frac{\partial v}{\partial y}=0, \\
u \frac{\partial u}{\partial x}+v \frac{\partial u}{\partial y}=v \frac{\partial^{2} u}{\partial y^{2}}-\frac{v}{k} u, \\
\rho c_{p}\left(u \frac{\partial T}{\partial x}+v \frac{\partial T}{\partial y}\right)=\frac{\partial}{\partial y}\left(\kappa \frac{\partial T}{\partial y}\right)-\frac{\partial q_{r}}{\partial y}
\end{gathered}
$$

with the following boundary conditions

$$
\begin{aligned}
\text { at } y & =0, \quad u=U_{w}(x)=-c e^{x / L}, \quad v=V_{w}(x)=v_{o} e^{x / 2 L}, \\
T & =T_{w}(x)=T_{\infty}+T_{o} e^{x / 2 L} \quad \text { and at } y \longrightarrow \infty, \\
u & \longrightarrow 0, \quad T \longrightarrow T_{\infty},
\end{aligned}
$$

where $u, v$ are the velocity components along $x$ and $y$ directions, respectively, $k$ is the permeability, $c_{p}$ is the specific heat at constant pressure, $v$ is the kinematic viscosity, $\rho$ is the density, and $T, \mu$, and $\kappa$ are the temperature, viscosity and thermal conductivity of the fluid, respectively. Further, $L$ is the characteristic length, $T_{w}$ is the variable temperature at the sheet, $T_{0}$ is the constant reference temperature, and $T_{\infty}$ is the constant free stream temperature. $U_{w}$ and $V_{w}$ are the shrinking velocity of the sheet and mass transfer velocity, respectively, where $c>0$ is the shrinking constant and $v_{o}$ is a constant (where $v_{o}<0$ corresponds to mass suction).

\section{Similarity Transformations}

We now introduce the stream function $\Psi(x, y)$ as

$$
u=\frac{\partial \psi}{\partial y}, \quad v=-\frac{\partial \psi}{\partial x} .
$$

Thus (1) is identically satisfied and the similarity transformations can be written as

$$
\begin{gathered}
\psi=\sqrt{2 v L c} f(\eta) e^{x / 2 L}, \quad \eta=y \sqrt{\frac{c}{2 v L}} e^{x / 2 L}, \\
\theta=\frac{T-T_{\infty}}{T_{w}-T_{\infty}} .
\end{gathered}
$$

On using (5) and (6) we obtain the expressions for velocity components in nondimensional form as

$$
u=c f^{\prime}(\eta) e^{x / L}, \quad v=-\sqrt{\frac{v c}{2 L}}\left(\eta f^{\prime}(\eta)+f(\eta)\right) e^{x / 2 L} .
$$


In order to obtain the similarity solutions, it is assumed that the permeability $k$ of the porous medium takes the following form

$$
k(x)=2 k_{o} e^{-x / L},
$$

where $k_{o}$ is reference permeability.

Following Chiam [42], thermal conductivity of the fluid is assumed to vary with temperature in a linear fashion as

$$
\kappa=\kappa_{\infty}(1+\varepsilon \theta),
$$

where $\varepsilon$ is the thermal conductivity variation parameter. In general $\varepsilon>0$ for fluids such as water and air, while $\varepsilon<0$ for fluids such as lubrication oils. The radiative heat flux in the energy equation is presumed to follow Rosseland approximation and is given by

$$
q_{r}=-\frac{4 \sigma_{1}}{3 k_{1}} \frac{\partial T^{4}}{\partial y}
$$

where $\sigma_{1}$ is the Stephan-Boltzmann constant and $k_{1}$ is the mean absorption constant. It is further assumed that the temperature difference within the fluid is sufficiently small so that $T^{4}$ may be expressed as a linear function of temperature $T$. This is done by expanding $T^{4}$ in a Taylor series about $T_{\infty}$ and omitting higher-order terms to yield

$$
T^{4} \cong 4 T_{\infty}^{3} T-3 T_{\infty}^{4} .
$$

The momentum (2) and the energy (3) thus reduce to the following nondimensional form

$$
\begin{gathered}
f^{\prime \prime \prime}+f f^{\prime \prime}-2 f^{\prime 2}-\frac{f^{\prime}}{K}=0, \\
\left(1+\frac{4 N}{3}\right) \theta^{\prime \prime}+\varepsilon \theta \theta^{\prime \prime}+\varepsilon \theta^{\prime 2}+\operatorname{Pr}\left(f \theta^{\prime}-f^{\prime} \theta\right)=0
\end{gathered}
$$

with the boundary conditions

$$
\begin{gathered}
\eta \longrightarrow 0: f^{\prime}(\eta)=-1, \quad f(\eta)=\frac{-v_{o}}{\sqrt{v c / 2 L}}=S, \quad \theta(\eta)=1, \\
\eta \longrightarrow \infty: f^{\prime}(\eta) \longrightarrow 0, \quad \theta(\eta) \longrightarrow 0
\end{gathered}
$$

where

$$
K=\frac{c k_{o}}{L v}, \quad \operatorname{Pr}=\frac{\mu c_{p}}{\kappa_{\infty}}, \quad N=\frac{4 \sigma_{1} T_{\infty}^{3}}{3 k_{1} \kappa_{\infty}}
$$

denote the permeability parameter, Prandtl number, and radiation parameter, respectively.

\section{Numerical Solution}

The coupled and highly nonlinear boundary value problem (BVP) described by (12) and (13) is not amenable to analytic closed form solution, therefore we resorted to numerical solution. The BVP has been solved by fourth-order RungeKutta scheme together with shooting method. The essence of shooting method to solve a BVP is to first convert the BVP into system of initial value problems and make suitable guesses for the unknown quantities such that the end conditions of the solution space are satisfied.

By making some transformations the BVP is reduced to the following system of initial value problems

$$
\begin{gathered}
f^{\prime}=p, \quad p^{\prime}=q, \quad q^{\prime}=2 p^{2}-f q+\frac{p}{K}, \\
\theta^{\prime}=z, \quad z^{\prime}=\frac{\operatorname{Pr}\left(f^{\prime} \theta-f z\right)-\varepsilon z^{2}}{1+(4 N / 3)+\varepsilon \theta}
\end{gathered}
$$

under the boundary conditions

$$
\begin{gathered}
f(0)=S, \quad p(0)=-1, \quad q(0)=r_{1}, \\
\theta(0)=1, \quad z(0)=r_{2} .
\end{gathered}
$$

Here $r_{1}=f^{\prime \prime}(0)$ and $r_{2}=\theta^{\prime}(0)$ are the initial guesses which are arbitrarily chosen and an iterative procedure is set to obtain solutions through Runge-Kutta fourth-order method. The computational procedure involved two challenges, firstly determination of $\eta_{\infty}$, that is, maximum value of $\eta$ for which $f^{\prime}(\eta) \rightarrow 0$ and $\theta(\eta) \rightarrow 0$ at $\eta \rightarrow \infty$ and secondly proper estimates for the unknown quantities $f^{\prime \prime}(0)$ and $\theta^{\prime}(0)$. It is not out of place to make remark that the "guesses" were made purely on hit and trial basis and their refinement was interpolated iteratively with the prescribed error tolerance. A grid independence study was also carried out to examine the effect of step size $\Delta \eta$ and the edge of the boundary layer $\eta_{\infty}$ on the solution in the quest for their optimization. The $\eta_{\max }$, that is, $\eta$ at infinity was so chosen that further changes in it do not produce changes in the values of $f^{\prime \prime}(0)$ and $\theta^{\prime}(0)$ (constant till $10^{-7}$ ) vis a vis boundary conditions are satisfied. A step size of $\Delta \eta=0.01$ was found to be satisfactory for a convergence criterion of $10^{-7}$ in all cases.

\section{Results and Discussion}

The numerical computations carried out for different sets of values of the parameters entering into the problem have been depicted through graphs and tables. The analysis, besides the effects of parameters on flow and heat transfer, also aims to compute the range of mass suction for parameter-values required for laminar flow on the shrinking sheet. Here we are inclined to recall the works of Miklavčič and Wang [20] and Fang and Zhang [24] who discussed flow due to linear shrinking sheet in clear Newtonian fluid. They reported that to facilitate the steady flow due to shrinking sheet mass suction parameter of strength greater or equal to 2 is required. Recently, Bhattacharyya [31] computed values of suction velocity for the flow due to exponentially shrinking sheet in clear fluid regime $(K \rightarrow \infty)$ and reported that laminar flow does not exist if the suction velocity is less than 2.266684 . Here it is emphasized that due to exponential shrinking of the sheet, the vorticity grows rapidly in the boundary layer and tends to go beyond the boundary layer, hence an adequate mass suction is required for existence of the laminar flow. Further, here it is worth noting that in the present analysis the sheet is placed at the bottom of porous medium 
which offers resistance to fluid traversal inside the porous matrix and contributes in some way in fluid stability, hence one may expect rather low suction rate as compared to the case of clear fluid situation. In present investigation, we did rigorous computations to extract the minimum suction rate required for ensuring the laminar flow in porous medium due to exponentially shrinking sheet. We found that in case of the porous medium one requires lesser suction values $(S \leq 2.266684$ when $K \neq \infty$ ) to contain the vorticity in the boundary layer depending upon the porous material chosen (see Table 1). The table shows that similarity solution exists for the exponential shrinking sheet placed at the bottom of the fluid saturated porous medium if $S \geq 2.18711$ when $K=$ $10 ; S \geq 2.22717$ when $K=20$. Further it is emphasised that when $K \rightarrow \infty$, then the present problem as far as momentum equation is concerned reduces to that of Bhattacharyya [31]. We see that when $K \rightarrow \infty$, then it is found that $S \geq$ 2.266662. This is in perfect agreement with Bhattacharyya [31]. Further, Miklavčič and Wang [20] observed that in flow problems involving shrinking surfaces, one may encounter nonunique solutions. In the present analysis we get dual solution regime. These solutions are closely dependent on mass suction velocity at the surface.

In order to solve the system of initial value problems we required proper estimates for the unknown quantities $f^{\prime \prime}(0)$ and $\theta^{\prime}(0)$. A large number of guesses for $f^{\prime \prime}(0)$ were tested for different values of permeability parameter $K$. These guesses were chosen on purely hit and trial basis such that the end conditions got satisfied. An error tolerance of magnitude $10^{-7}$ was prescribed for the computations. Tables 2, 3, 4, and 5 display rigorously computed values of $f^{\prime \prime}(0)$ for dual solution regime for momentum equation and the range of mass suction for various set of values of permeability parameter.

The profiles for the skin friction coefficient $f^{\prime \prime}(0)$ and rate of heat transfer $-\theta^{\prime}(0)$ versus suction velocity parameter $S$ drawn for various values of permeability parameter $K$ are shown in Figures 1 and 2, respectively. We find dual solution regimes for both $f^{\prime \prime}(0)$ and $-\theta^{\prime}(0)$ for the different values of suction velocity parameter. Further Figure 1 depicts that the skin friction coefficient $f^{\prime \prime}(0)$ registers increase with an increase in suction velocity for the first solution whereas it decays in the case of second solution for increasing values of $S$. The figure also shows that with increasing values of permeability parameter $K, f^{\prime \prime}(0)$ for the first solution decays whereas for the second solution it increases. From Figure 2 we see that $-\theta^{\prime}(0)$ increases for the first solution with increase in $S$ whereas for the second solution it decreases. Moreover with the increasing values of the permeability parameter $K,-\theta^{\prime}(0)$ increases for the second solution but insignificant changes occur in case of first solution.

Figure 3 shows the variation in $f^{\prime}(\eta)$ with respect to the suction velocity parameter $S$. For the first solution $f^{\prime}(\eta)$ decreases numerically within the boundary layer for increasing values of suction parameter $S$ and becomes zero at the edge of the momentum boundary layer for every value $S$. The profiles of second solution exhibit a similar pattern.

The variation in $f^{\prime}(\eta)$ for different values of permeability parameter $K$, when the suction parameter is set as $S=2.4$

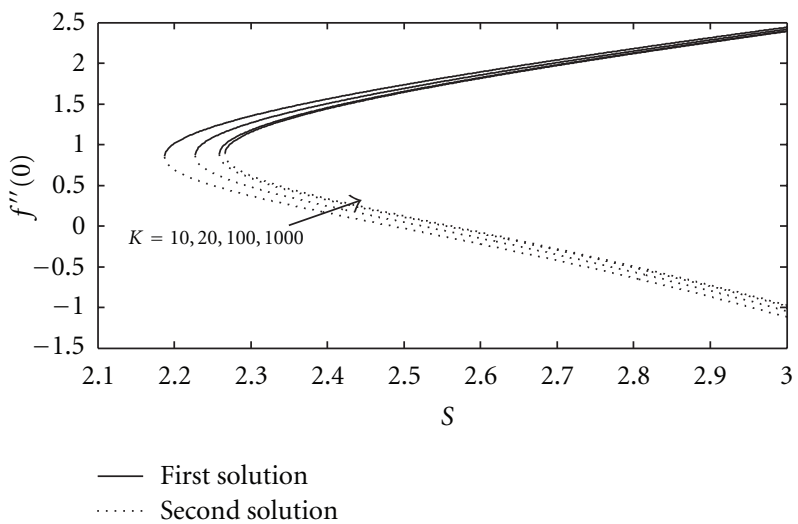

Figure 1: Profiles for skin friction coefficient $\left(f^{\prime \prime}(0)\right)$ for variation in permeability parameter $K$.

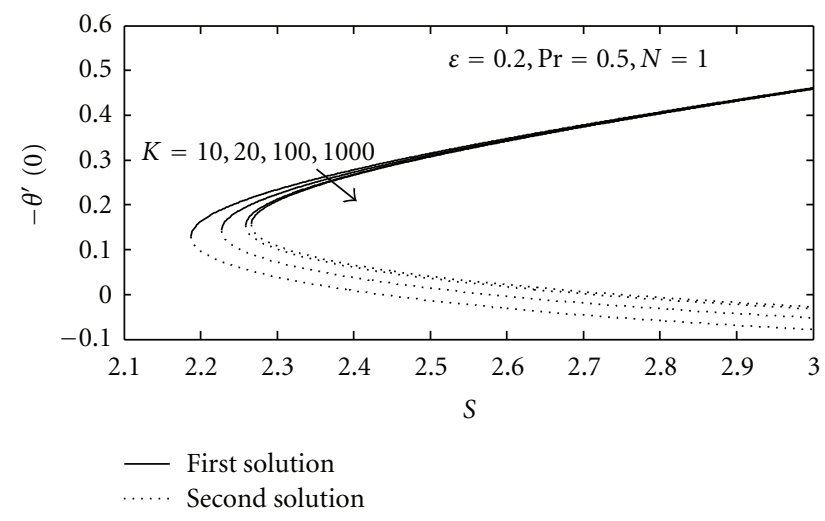

FIguRe 2: Profiles for temperature gradient at the sheet $\left(-\theta^{\prime}(0)\right)$ for variation in permeability parameter $K$.

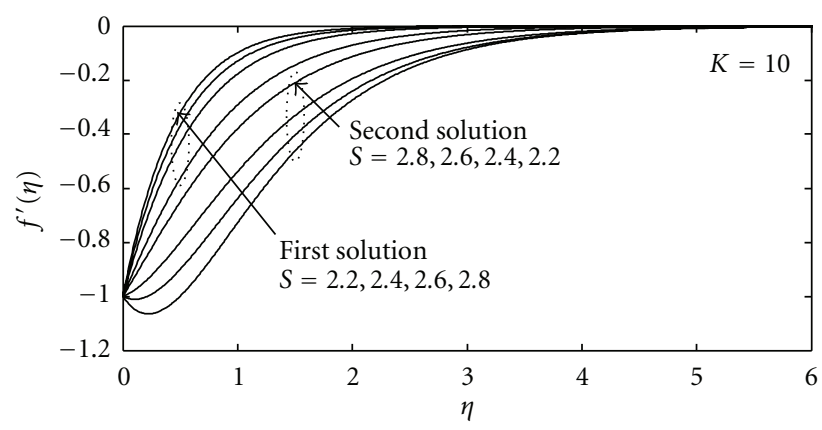

Figure 3: Dual velocity $\left(f^{\prime}(\eta)\right)$ profiles for variation in suction velocity $S$.

is shown in Figure 4. We see that for the first solution $f^{\prime}(\eta)$ increases numerically with the increasing values of $K$ whereas $f^{\prime}(\eta)$ decreases in the case of second solution for the increasing values of $K$. The figure also reveals that effect of permeability is more significant on the second solution as compared to the first solution.

Variations in shear stress $f^{\prime \prime}(\eta)$ in the boundary layer region for the suction parameter values are shown in Figure 5. For the first solution, shear stress in the vicinity 
TABLE 1: Variation of minimum suction velocity $S_{s}$ required for flow phenomena with the permeability parameter $K$.

\begin{tabular}{lcc}
\hline Permeability parameter $K$ & Minimum suction velocity $S=S_{s}$ & Bhattacharyya [31] \\
\hline 10 & Present paper & - \\
20 & 2.18711 & - \\
100 & 2.22717 & - \\
1000 & 2.258819 & - \\
50,000 & 2.26590 & 2.266684 \\
\hline
\end{tabular}

TABLE 2: Estimates for guesses of $f^{\prime \prime}(0)$ for both the solutions of the velocity profile when $K=10$.

\begin{tabular}{|c|c|c|c|}
\hline \multicolumn{2}{|c|}{ First solution } & \multicolumn{2}{|c|}{ Second solution } \\
\hline Range of suction velocity $(S)$ & Guesses for $f^{\prime \prime}(0)$ & Range of suction velocity $(S)$ & Guesses for $f^{\prime \prime}(0)$ \\
\hline $2.18711 \leq S \leq 2.200$ & $.9,1$ & $2.18711 \leq S \leq 2.225$ & $.6, .7$ \\
\hline $2.200 \leq S \leq 2.250$ & $1,1.2$ & $2.225 \leq S \leq 2.251$ & $.5, .6$ \\
\hline $2.250 \leq S \leq 2.290$ & $1.2,1.3$ & $2.251 \leq S \leq 2.295$ & $.4, .44$ \\
\hline $2.290 \leq S \leq 2.335$ & $1.3,1.4$ & $2.295 \leq S \leq 2.335$ & $.3, .35$ \\
\hline $2.335 \leq S \leq 2.385$ & $1.4,1.5$ & $2.335 \leq S \leq 2.350$ & $.22, .3$ \\
\hline $2.385 \leq S \leq 2.440$ & $1.5,1.6$ & $2.350 \leq S \leq 2.385$ & $.21, .23$ \\
\hline $2.440 \leq S \leq 2.499$ & $1.6,1.7$ & $2.385 \leq S \leq 2.395$ & $.19, .2$ \\
\hline $2.499 \leq S \leq 2.560$ & $1.7,1.8$ & $2.395 \leq S \leq 2.421$ & $.1, .11$ \\
\hline $2.560 \leq S \leq 2.620$ & $1.8,1.9$ & $2.421 \leq S \leq 2.460$ & $.05, .09$ \\
\hline $2.620 \leq S \leq 2.700$ & $1.9,2.0$ & $2.460 \leq S \leq 2.474$ & $0, .05$ \\
\hline $2.700 \leq S \leq 2.774$ & $2,2.1$ & $2.474 \leq S \leq 2.500$ & $0,-.05$ \\
\hline $2.774 \leq S \leq 2.850$ & $2.1,2.2$ & $2.500 \leq S \leq 2.540$ & $-.1,-.05$ \\
\hline $2.850 \leq S \leq 2.914$ & $2.1,2.3$ & $2.540 \leq S \leq 2.570$ & $-.1,-.15$ \\
\hline \multirow[t]{13}{*}{$2.914 \leq S \leq 3.010$} & $2.3,2.4$ & $2.570 \leq S \leq 2.590$ & $-.2,-.15$ \\
\hline & & $2.590 \leq S \leq 2.620$ & $-.2,-.25$ \\
\hline & & $2.620 \leq S \leq 2.640$ & $-.3,-.25$ \\
\hline & & $2.640 \leq S \leq 2.670$ & $-.3,-.35$ \\
\hline & & $2.670 \leq S \leq 2.690$ & $-.35,-.4$ \\
\hline & & $2.690 \leq S \leq 2.722$ & $-.4,-.45$ \\
\hline & & $2.722 \leq S \leq 2.770$ & $-.5,-.55$ \\
\hline & & $2.770 \leq S \leq 2.790$ & $-.55,-.6$ \\
\hline & & $2.790 \leq S \leq 2.833$ & $-.6,-.7$ \\
\hline & & $2.833 \leq S \leq 2.875$ & $-.7,-.8$ \\
\hline & & $2.875 \leq S \leq 2.920$ & $-.8,-.9$ \\
\hline & & $2.920 \leq S \leq 2.960$ & $-.9,-1$ \\
\hline & & $2.960 \leq S \leq 3.001$ & $-1,-1.1$ \\
\hline
\end{tabular}

TABLE 3: Estimates for guesses of $f^{\prime \prime}(0)$ for both the solutions of the velocity profile when $K=20$.

\begin{tabular}{lccc}
\hline & \multicolumn{1}{c}{$\begin{array}{c}\text { Sirst solution } \\
\text { Range of suction velocity }(S)\end{array}$} & Second solution \\
\hline $2.22717 \leq S \leq 2.270$ & $1,1.1$ & Range of suction velocity $(S)$ & Guesses for $f^{\prime \prime}(0)$ \\
$2.270 \leq S \leq 2.350$ & $1.3,1.4$ & $2.22717 \leq S \leq 2.300$ & $.5, .55$ \\
$2.350 \leq S \leq 2.483$ & $1.5,1.6$ & $2.300 \leq S \leq 2.399$ & $.3, .35$ \\
$2.483 \leq S \leq 2.600$ & $1.7,1.8$ & $2.399 \leq S \leq 2.444$ & $.1, .15$ \\
$2.600 \leq S \leq 2.700$ & $1.9,2.0$ & $2.444 \leq S \leq 2.548$ & $0,0.05$ \\
$2.700 \leq S \leq 2.890$ & $2.1,2.2$ & $2.548 \leq S \leq 2.600$ & $-.05,-.1$ \\
$2.890 \leq S \leq 3.000$ & $2.3,2.4$ & $2.600 \leq S \leq 2.700$ & $-.25,-.3$ \\
& & $2.700 \leq S \leq 2.802$ & $-.45,-.5$ \\
& & $2.802 \leq S \leq 2.904$ & $-.70,-.75$ \\
\end{tabular}


TABLE 4: Estimates for guesses of $f^{\prime \prime}(0)$ for both the solutions of the velocity profile when $K=100$.

\begin{tabular}{|c|c|c|c|}
\hline \multicolumn{2}{|c|}{ First solution } & \multicolumn{2}{|c|}{ Second solution } \\
\hline Range of suction velocity $(S)$ & Guesses for $f^{\prime \prime}(0)$ & Range of suction velocity $(S)$ & Guesses for $f^{\prime \prime}(0)$ \\
\hline $2.258819 \leq S \leq 2.415$ & $1.3,1.4$ & $2.258819 \leq S \leq 2.350$ & $.5, .6$ \\
\hline $2.415 \leq S \leq 2.460$ & $1.4,1.5$ & $2.350 \leq S \leq 2.420$ & $.3, .5$ \\
\hline $2.460 \leq S \leq 2.500$ & $1.5,1.6$ & $2.420 \leq S \leq 2.515$ & $.1, .3$ \\
\hline $2.500 \leq S \leq 2.570$ & $1.6,1.7$ & $2.515 \leq S \leq 2.670$ & $-.1,-.11$ \\
\hline $2.570 \leq S \leq 2.620$ & $1.7,1.8$ & $2.670 \leq S \leq 2.745$ & $-.2,-.3$ \\
\hline $2.620 \leq S \leq 2.700$ & $1.8,1.9$ & $2.745 \leq S \leq 2.870$ & $-.5,-.55$ \\
\hline $2.700 \leq S \leq 2.755$ & $1.9,2.0$ & $2.870 \leq S \leq 2.880$ & $-.5,-.6$ \\
\hline $2.755 \leq S \leq 2.890$ & $2.1,2.2$ & $2.880 \leq S \leq 3.001$ & $-.9,-.8$ \\
\hline $2.890 \leq S \leq 3.011$ & $2.3,2.4$ & & \\
\hline
\end{tabular}

TABLE 5: Estimates for guesses of $f^{\prime \prime}(0)$ for both the solutions of the velocity profile when $K=1000$.

\begin{tabular}{lccc}
\hline \multicolumn{1}{c}{$\begin{array}{c}\text { First solution } \\
\text { Range of suction velocity }(S)\end{array}$} & Guesses for $f^{\prime \prime}(0)$ & \multicolumn{2}{c}{$\begin{array}{c}\text { Second solution } \\
\text { Range of suction velocity }(S)\end{array}$} \\
\hline $2.26590 \leq S \leq 2.290$ & $.9, .1$ & $2.26590 \leq S \leq 2.400$ & $.4, .5$ \\
$2.290 \leq S \leq 2.470$ & $1.4,1.5$ & $2.400 \leq S \leq 2.540$ & $.1, .2$ \\
$2.470 \leq S \leq 2.640$ & $1.7,1.8$ & $2.540 \leq S \leq 2.710$ & $-.1,-.2$ \\
$2.640 \leq S \leq 2.850$ & $2,2.1$ & $2.710 \leq S \leq 2.850$ & $-.4,-.5$ \\
$2.850 \leq S \leq 3.055$ & $2.3,2.4$ & $2.850 \leq S \leq 3.000$ & $-.8,-.9$ \\
\hline
\end{tabular}

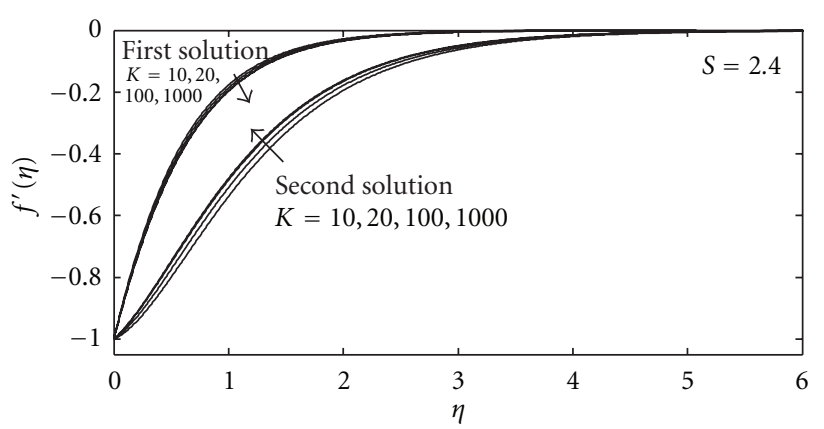

Figure 4: Dual velocity $\left(f^{\prime}(\eta)\right)$ profiles for variation in permeability parameter $K$.

of the wall increases with the increasing values of suction parameter $S$ but at some critical distance from the wall, $f^{\prime \prime}(\eta)$ decays with the increasing values of $S$. However, opposite trends in $f^{\prime \prime}(\eta)$ are observed in the case of second solution for increasing values of $S$.

Figure 6 depicts the effect of permeability parameter $K$ on shear stress $f^{\prime \prime}(\eta)$ in the boundary layer. We observe that for the first solution, $f^{\prime \prime}(\eta)$ decreases near the wall for the increasing values of $K$. For the second solution, $f^{\prime \prime}(\eta)$ increases with increasing $K$ near the wall. However, variations in $f^{\prime \prime}(\eta)$ are reversed for variation in $K$ in both the solutions at a certain distance from the wall. The figure also reveals that effect of $K$ on $f^{\prime \prime}(\eta)$ is more pronounced for the second solution as compared to that of the first one.

Figure 7 exhibits temperature profiles for various suction values. It is clear from the figure that the temperature $\theta(\eta)$ for

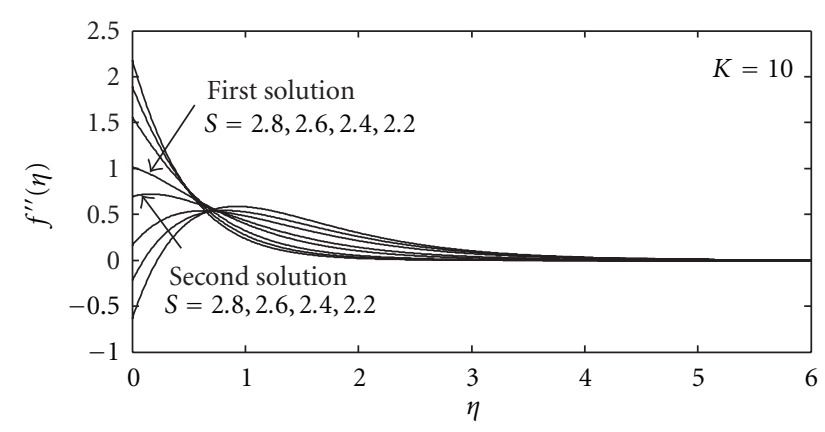

FIGURE 5: Shear stress $\left(f^{\prime \prime}(\eta)\right)$ profiles for variation in suction velocity $S$.

the first solution decays with the increasing values of suction parameter $S$ whereas in the second solution case we find that $\theta(\eta)$ increases with the increasing values of $S$. We also observe that for the same set of suction values, the thermal boundary layer is thicker for the second solution compared to that of first solution.

Figure 8 displays variation in temperature for varying values of permeability parameter $K$. We see that the effect of permeability $K$ on $\theta(\eta)$ is insignificant for the first solution whereas permeability has pronounced effect on temperature for second solution.

Figure 9 shows the variation of temperature $\theta(\eta)$ for different values of the Prandtl number Pr. We see that for both solutions, $\theta(\eta)$ decays with the increasing Pr values. However it is interesting to note that thermal boundary layer for 


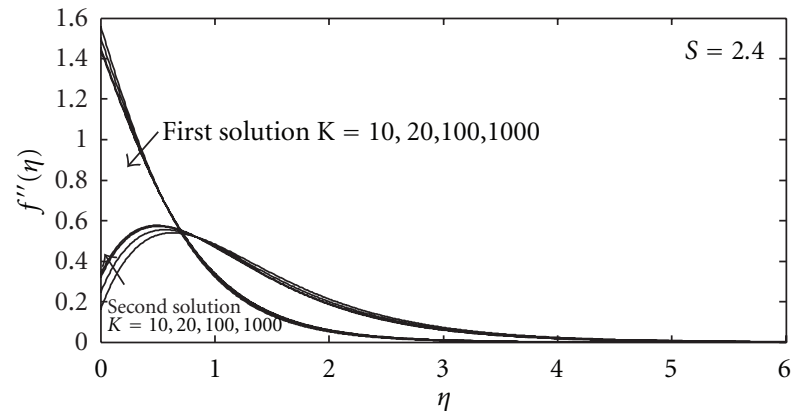

Figure 6: Shear stress $\left(f^{\prime \prime}(\eta)\right)$ profiles for variation in permeability parameter $K$.

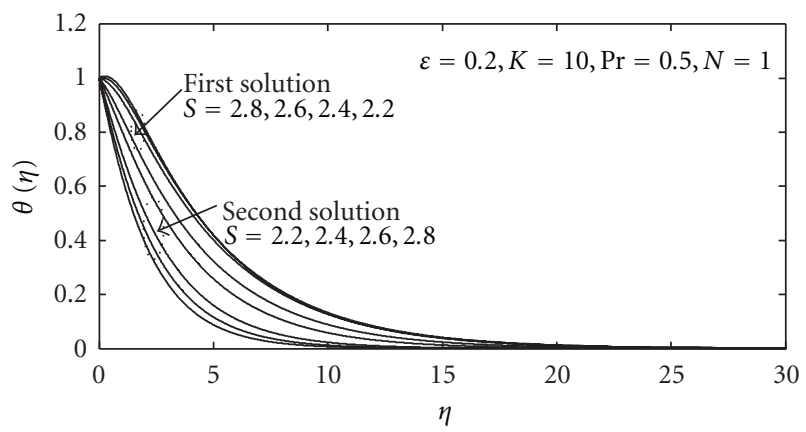

Figure 7: Temperature $(\theta(\eta))$ profiles for variation in suction velocity $S$.

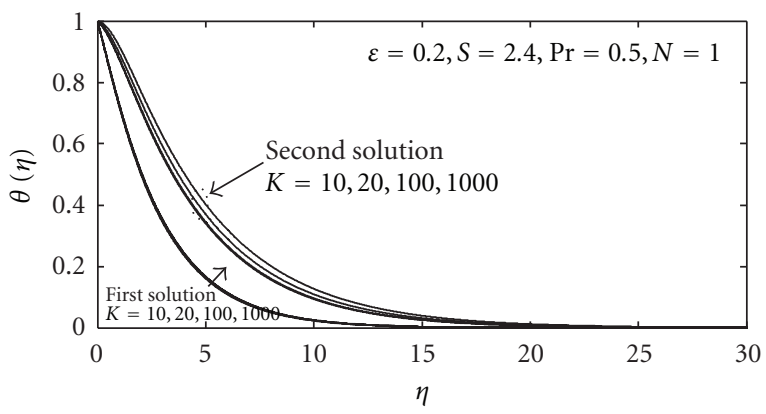

Figure 8: Dual temperature $(\theta(\eta))$ profiles for variation in permeability parameter $K$.

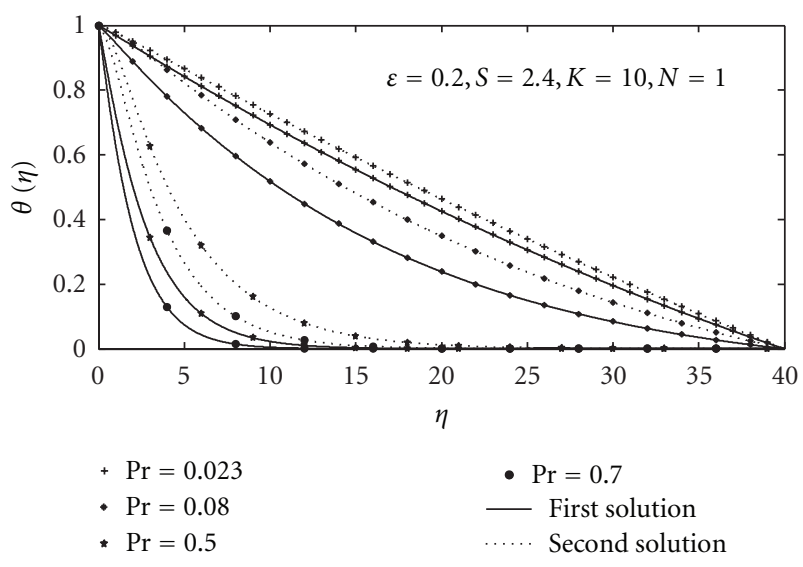

Figure 9: Dual temperature $(\theta(\eta))$ profiles for variation in Prandtl number Pr.

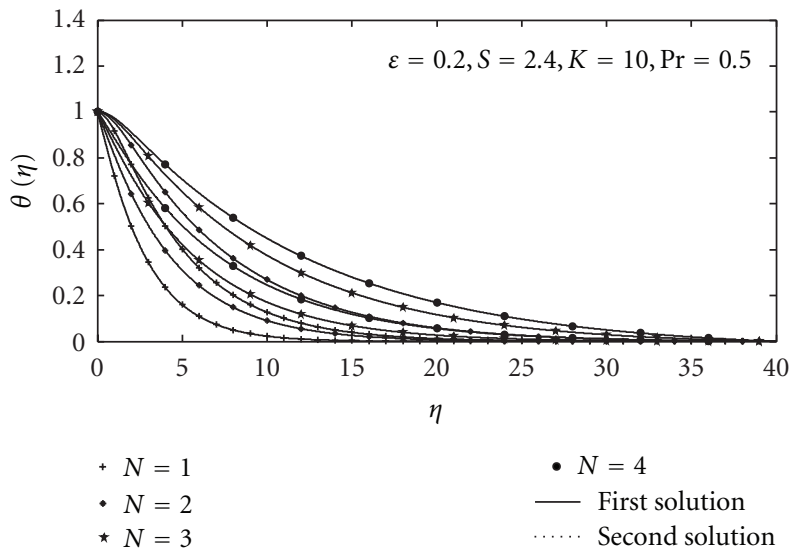

FIGURE 10: Dual temperature $(\theta(\eta))$ profiles for variation in radiation parameter $N$.

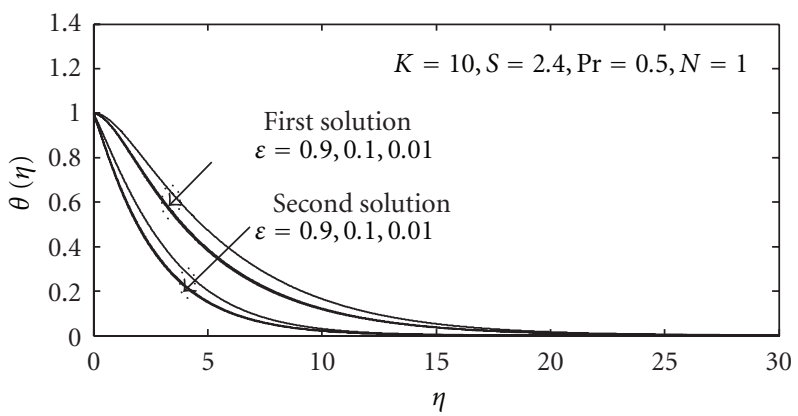

Figure 11: Dual temperature $(\theta(\eta))$ profiles for variation in the parameter $\varepsilon$.

the first solution is thicker as compared to that of second solution for all Pr values under consideration.

Prandtl number physically characterizes relative importance of momentum diffusion to thermal diffusion in the flow field. Low density fluids such as liquid metals (e.g., mercury, $\operatorname{Pr}=0.023$ ) enjoy low Prandtl number values, that is, less than unity. In thermal regime with low Prandtl number values heat diffuses at faster rate. On the contrary, oil having low thermal conductivity (high Pr values) gives rise to thinner boundary layer.

The effect of the radiation parameter $N$ on $\theta(\eta)$ is shown in Figure 10. The radiation parameter $N$ is reciprocal of Stark number (also known as Stephan number) which measures the relative importance of thermal radiation transfer to the conduction heat transfer. Thus larger values of $N$ mean dominance of thermal radiation over conduction. Consequently, larger values of $N$ indicate that larger amount of radiative heat energy is poured into the flow field, causing rise in $\theta(\eta)$. We conclude from the figure that increase in radiation parameter results in an increase in fluid temperature.

Figure 11 displays the effect of thermal conductivity perturbation parameter $\varepsilon$ on temperature. It is observed that $\theta(\eta)$ increases with an increase in parameter $\varepsilon$. 


\section{Acknowledgment}

The authors are thankful to the learned referees for offering their valuable suggestions to improve the paper.

\section{References}

[1] L. J. Crane, "Flow past a stretching plate," Zeitschrift für Angewandte Mathematik und Physik ZAMP, vol. 21, no. 4, pp. 645647,1970

[2] S. J. Liao, "On the analytic solution of magnetohydrodynamic flows of non-Newtonian fluids over a stretching sheet," Journal of Fluid Mechanics, no. 488, pp. 189-212, 2003.

[3] W. A. Khan and I. Pop, "Boundary-layer flow of a nanofluid past a stretching sheet," International Journal of Heat and Mass Transfer, vol. 53, no. 11-12, pp. 2477-2483, 2010.

[4] A. Ishak, R. Nazar, and I. Pop, "Mixed convection stagnation point flow of a micropolar fluid towards a stretching sheet," Meccanica, vol. 43, no. 4, pp. 411-418, 2008.

[5] K. R. Rajgopal, T. Y. Na, and A. S. Gupta, "Flow of a viscoelastic fluid over a stretching sheet," RheolActa, vol. 23, pp. 213-215, 1984.

[6] P. H. Veena, S. Abel, K. Rajagopal, and V. K. Pravin, "Heat transfer in a visco-elastic fluid past a stretching sheet with viscous dissipation and internal heat generation," Zeitschrift fur Angewandte Mathematik und Physik, vol. 57, no. 3, pp. 447463, 2006.

[7] K. Ahmad, R. Nazar, A. Ishak, and I. Pop, "Unsteady threedimensional boundary layer flow due to a stretching surface in a micropolar fluid," International Journal for Numerical Methods in Fluids, vol. 28, pp. 118-122, 2011.

[8] H. I. Andersson and O. A. Valnes, "Flow of a heated ferrofluid over a stretching sheet in the presence of a magnetic dipole," Acta Mechanica, vol. 128, no. 1-2, pp. 39-47, 1998.

[9] D. S. Chauhan and R. Agrawal, "MHD flow through a porous medium adjacent to a stretching sheet: numerical and an approximate solution," European Physical Journal Plus, vol. 126, no. 5, pp. 1-14, 2011.

[10] S. Liao, "A new branch of solutions of boundary-layer flows over an impermeable stretched plate," International Journal of Heat and Mass Transfer, vol. 48, no. 12, pp. 2529-2539, 2005.

[11] S. J. Liao, "A new branch of solutions of boundary-layer flows over a permeable stretching plate," International Journal of Non-Linear Mechanics, vol. 42, no. 6, pp. 819-830, 2007.

[12] E. Magyari and B. Keller, "Heat and mass transfer in the boundary layers on an exponentially stretching continuous surface," Journal of Physics D, vol. 32, no. 5, pp. 577-585, 1999.

[13] E. M. A. Elbashbeshy, "Heat transfer over an exponentially stretching continuous surface with suction," Archives of Mechanics, vol. 53, no. 6, pp. 643-651, 2001.

[14] R. A. M. Q. Al-Odat, T. A. Damesh, and T. A. Al-Azab, "Thermal boundary layer on an exponentially stretching continuous surface in the presence of magnetic field effect," International Journal of Applied Mechanics and Engineering, vol. 11, pp. 289-299, 2006.

[15] M. K. Partha, P. V. S. N. Murthy, and G. P. Rajasekhar, "Effect of viscous dissipation on the mixed convection heat transfer from an exponentially stretching surface," Heat and Mass Transfer, vol. 41, no. 4, pp. 360-366, 2005.

[16] E. Sanjayanand and S. K. Khan, "On heat and mass transfer in a viscoelastic boundary layer flow over an exponentially stretching sheet," International Journal of Thermal Sciences, vol. 45, no. 8, pp. 819-828, 2006.
[17] S. K. Khan and E. Sanjayanand, "Viscoelastic boundary layer flow and heat transfer over an exponential stretching sheet," International Journal of Heat and Mass Transfer, vol. 48, no. 8, pp. 1534-1542, 2005.

[18] M. Sajid and T. Hayat, "Influence of thermal radiation on the boundary layer flow due to an exponentially stretching sheet," International Communications in Heat and Mass Transfer, vol. 35, no. 3, pp. 347-356, 2008.

[19] C. Y. Wang, "Liquid film on an unsteady stretching sheet, Quart," Applied Mathematics, vol. 48, pp. 601-610, 1990.

[20] M. Miklavčič and C. Y. Wang, "Viscous flow due to a shrinking sheet," Quarterly of Applied Mathematics, vol. 64, no. 2, pp. 283-290, 2006.

[21] C. Y. Wang, "Stagnation flow towards a shrinking sheet," International Journal of Non-Linear Mechanics, vol. 43, no. 5, pp. 377-382, 2008.

[22] T. Hayat, Z. Abbas, and N. Ali, "MHD flow and mass transfer of a upper-convected Maxwell fluid past a porous shrinking sheet with chemical reaction species," Physics Letters, Section A, vol. 372, no. 26, pp. 4698-4704, 2008.

[23] T. Fang, "Boundary layer flow over a shrinking sheet with power-law velocity," International Journal of Heat and Mass Transfer, vol. 51, no. 25-26, pp. 5838-5843, 2008.

[24] T. Fang and J. Zhang, "Closed-form exact solutions of MHD viscous flow over a shrinking sheet," Communications in Nonlinear Science and Numerical Simulation, vol. 14, no. 7, pp. 2853-2857, 2009.

[25] T. G. Fang, J. Zhang, and S. S. Yao, "Viscous flow over an unsteady shrinking sheet with mass transfer," Chinese Physics Letters, vol. 26, no. 1, Article ID 014703, 2009.

[26] N. F. M. Noor, S. Awang Kechil, and I. Hashim, "Simple nonperturbative solution for MHD viscous flow due to a shrinking sheet," Communications in Nonlinear Science and Numerical Simulation, vol. 15, no. 2, pp. 144-148, 2010.

[27] T. Hayat, Z. Abbas, and M. Sajid, "On the analytic solution of magnetohydrodynamic flow of a second grade fluid over a shrinking sheet," Journal of Applied Mechanics, Transactions ASME, vol. 74, no. 6, pp. 1165-1171, 2007.

[28] T. Fang, W. Liang, and C. F. F. Lee, "A new solution branch for the Blasius equation-A shrinking sheet problem," Computers and Mathematics with Applications, vol. 56, no. 12, pp. 30883095, 2008.

[29] N. F. Mohd and I. Hashim, "MHD flow and heat transfer adjacent to a permeable shrinking sheet embedded in a porous medium," Sains Malaysiana, vol. 38, no. 4, pp. 559-565, 2009.

[30] D. S. Chauhan and R. Agrawal, "MHD flow and heat transfer in a channel bounded by a shrinking sheet and a plate with a porous substrate," Journal of Engineering Physics and Thermophysics, vol. 84, no. 5, pp. 1034-1046, 2011.

[31] K. Bhattacharyya, "Boundary layer flow and heat transfer over an exponentially shrinking sheet," Chinese Physics Letters, vol. 28, no. 7, Article ID 074701, 2011.

[32] B. S. Dandapat, B. Santra, and K. Vajravelu, "The effects of variable fluid properties and thermocapillarity on the flow of a thin film on an unsteady stretching sheet," International Journal of Heat and Mass Transfer, vol. 50, no. 5-6, pp. 991-996, 2007.

[33] P. Vyas and A. Rai, "Radiative flow with variable thermal conductivity over a noniIsothermal Stretching sheet in a porous medium, Int," Journal of Contemporary Mathematical Sciences, vol. 5, pp. 2685-2698, 2010. 
[34] U. Sarma and G. C. Hazarika, "Effects of variable viscosity and thermal conductivity on heat and mass transfer flow along a vertical plate in the presence of a magnetic field," Latin-American Journal of Physics Education, vol. 5, pp. 100-106, 2011.

[35] Y. Khan, Q. Wu, N. Faraz, and A. Yildirim, "The effects of variable viscosity and thermal conductivity on a thin film flow over a shrinking/stretching sheet," Computers and Mathematics with Applications, vol. 61, no. 11, pp. 3391-3399, 2011.

[36] P. Vyas and N. Srivastava, "Radiative MHD flow over a nonisothermal stretching sheet in a porous medium," Applied Mathematical Sciences, vol. 4, no. 49-52, pp. 2475-2484, 2010.

[37] P. Vyas and A. Ranjan, "Dissipative MHD boundary-layer flow in a porous medium over a sheet stretching nonlinearly in the presence of radiation," Applied Mathematical Sciences, vol. 4, no. 61-64, pp. 3133-3142, 2010.

[38] D. S. Chauhan and V. Kumar, "Radiation effects on unsteady flow through a porous medium channel with velocity and temperature slip boundary conditions," Applied Mathematical Sciences, vol. 6, no. 33-36, pp. 1759-1769, 2012.

[39] D. S. Chauhan and R. Agarwal, "MHD coupled flow and heat transfer across a porous layer due to an oscillating plate with radiation," Afrika Mathematica. In press.

[40] T. Hayat, S. A. Shehzad, M. Qasim, and A. Alsaedi, "Radiative flow with variable thermal conductivity in porous medium," Zeitschrift für Naturforschung, vol. 67, no. 3-4, pp. 153-159, 2012.

[41] E. M. A. Elbashbeshy and T. G. Emam, "Effects of thermal radiation and heat transfer over an unsteady stretching surface embedded in a porous medium in the presence of heat source or sink," Thermal Science, vol. 15, no. 2, pp. 477-485, 2011.

[42] T. C. Chiam, "Heat transfer in a fluid with variable thermal conductivity over a linearly stretching sheet," Acta Mechanica, vol. 129, no. 1-2, pp. 63-72, 1998. 

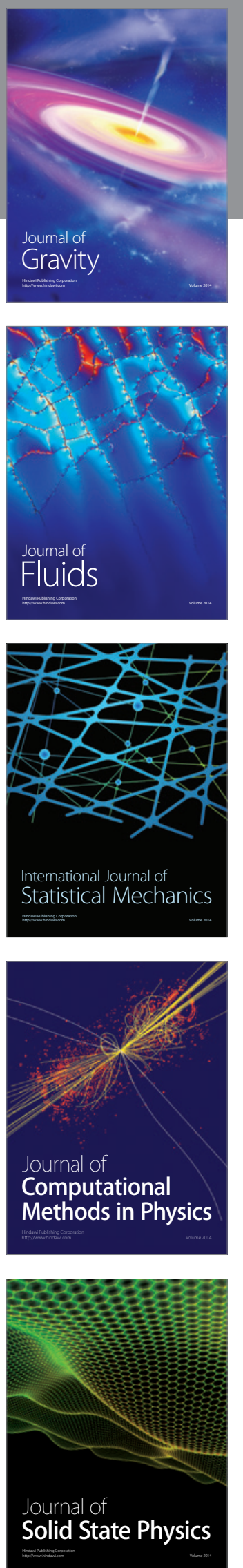
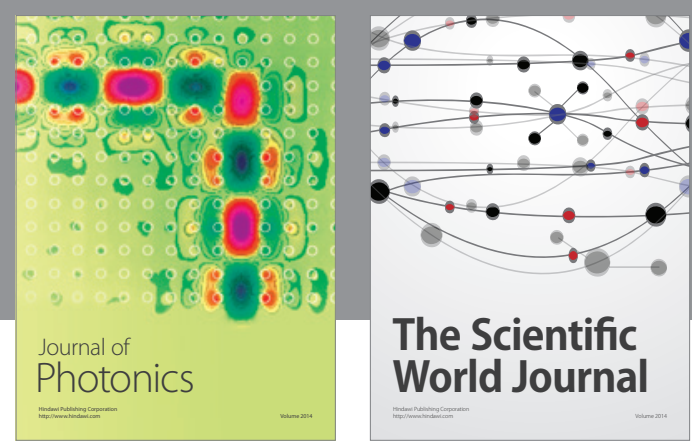

The Scientific World Journal

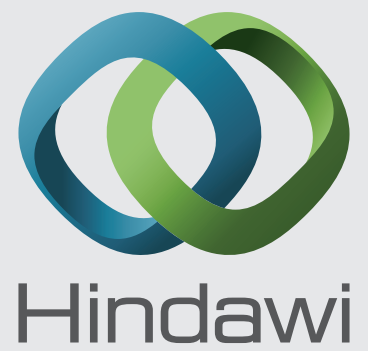

Submit your manuscripts at http://www.hindawi.com
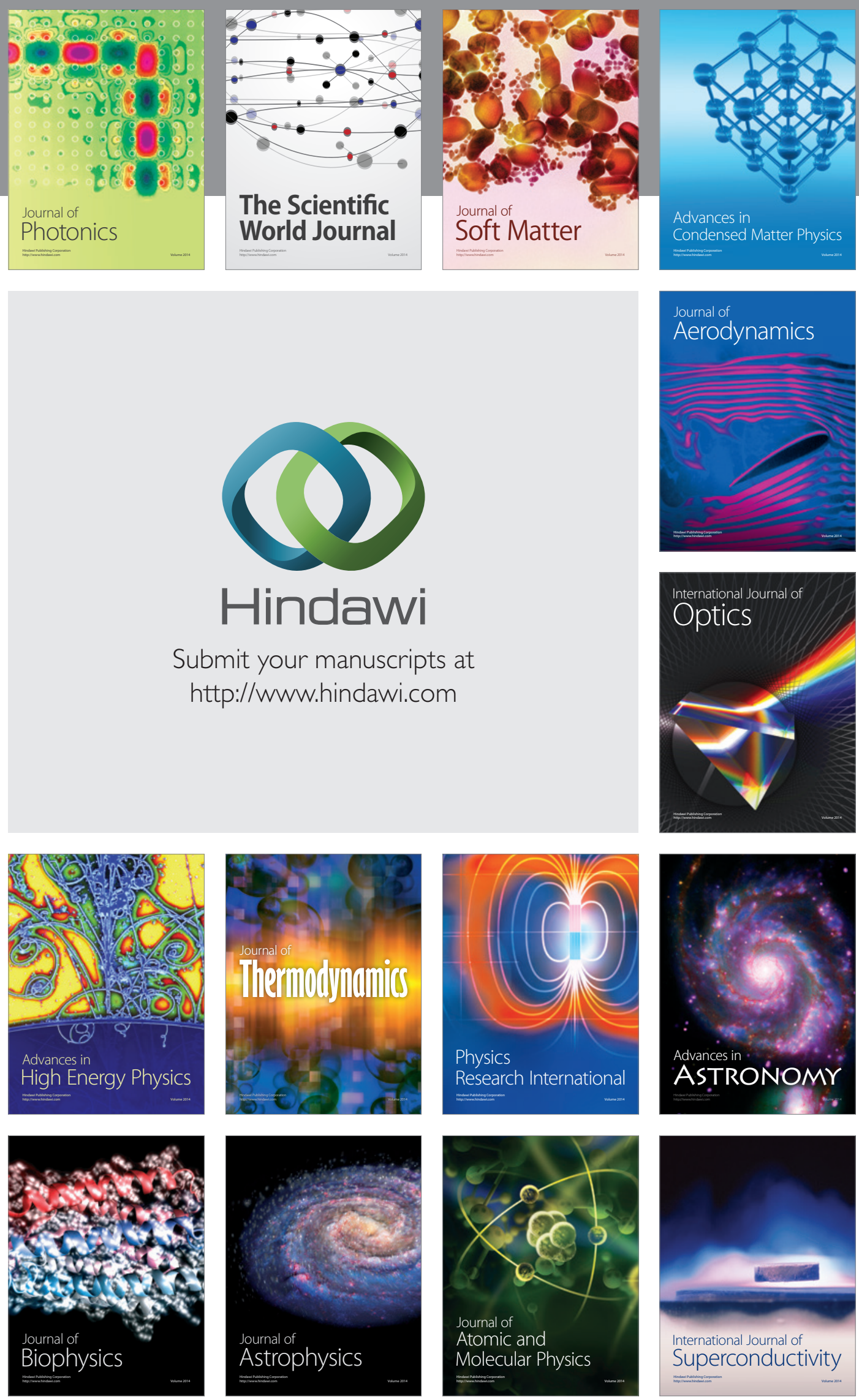
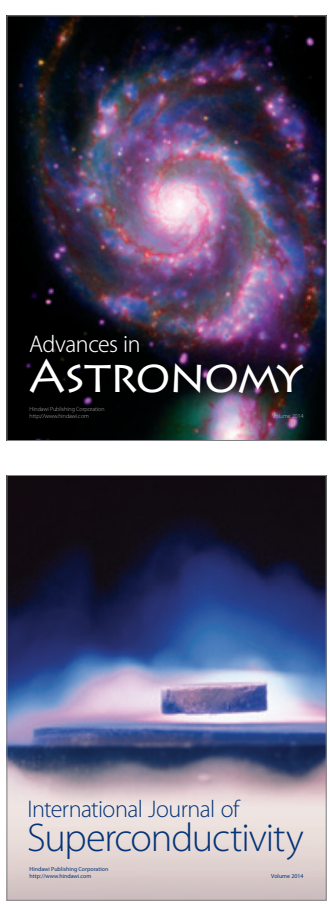